\title{
NUMERICAL ANALYSIS OF EXPERIMENTAL RESEARCH ON BUCKLING OF CLOSED SHALLOW CONICAL SHELLS UNDER EXTERNAL PRESSURE
}

\author{
Alexey Karasev, Maryna Varianychko, Yaroslav Bessmertnyi \\ Vasily Krasovsky, Gennady Karasev \\ Prydniprovs'ka State Academy of Civil Engineering and Architecture, Dnipro, Ukraine \\ e-mail:karasev.alexey@mail.ru; varianichko.mari@gmail.com; yaroslavbessmertnyi@gmail.com; \\ stmehanika@gmail.com; karasev.gennady@mail.ru
}

\begin{abstract}
Numerical analysis of experimental research on buckling of closed shallow conical shells under external pressure was carried out in ANSYS Inc simulation software. The obtained experimental data was compared with calculation results for more than 100 small-sized specimens of conical shells with a wide range of different geometry. All most important features of carrying out physical experiments were taken into account in the simulations. In particular, orthotropy of the shell material has been considered. The critical pressure values can be obtained in ANSYS software for existing conical shell structures with known geometry deviation comparing to ideal.
\end{abstract}

Keywords: conical shallow shell, buckling, external pressure, orthotropy, numerical analysis

\section{Formulation of the problem}

The problem of buckling of closed elastic shallow circular thin-walled cones under external pressure is a classic problem of theory of shells (Bushnell, 1981; Hoff and Singer, 1960; Seide, 1962; Singer, 1961). Both linear and geometrically nonlinear solutions were found for this problem. It is well known fact that sensitivity of critical and limit pressures to initial imperfections in the middle surface of shallow conical shells is relatively small. This is true in comparison with smooth longitudinally compressed thin-walled cylinders and spherical shells under external pressure (Grigolyuk and Kabanov, 1978; Volmir, 1967). The question of the influence of material orthotropy on the buckling load of shallow conical shells still remains insufficiently studied.

A series of experiments was carried in the first decade of the XXI century in Ukraine. These were experiments of buckling of small-sized shallow closed circular conical shell specimens (more than 100 specimens) in a wide range of variable geometry. The obtained experimental data was compared with calculation results. Calculations were carried out on the basis of different linear theories and calculation models for ideal shells. Calculations on the basis of the finite element method were done in ANSYS (Krasovsky et al., 2005a,b). Aforementioned researches demonstrated a number of insufficiently studied issues. These questions as a part of the problem are both of theoretical and applied engineering interest. The clarification of calculations is the current issue because studied shells are widely used as protecting elements and coatings for various tanks and for others devices in the chemical industry, shipbuilding, and missile and aircraft manufacturing. Moreover, conical shells are often used as object coverage of road network and bridge structures. This is confirmed by a number of modern papers devoted to this subject (Elishakoff, 2012; Krasovsky and Karasev, 2016; Maali et al., 2012).

The aim of the work is to evaluate the effectiveness of numerical analysis of buckling of closed shallow conical shells under external pressure, by comparing the calculation carried out in ANSYS simulation software with experiments, as was presented in (Krasovsky et al., 2005a,b), and which were made on small specimens. 


\section{Specimens, general relations and experimental data}

The research program included a test of 4 series of closed small conical shells (over 100 specimens) made of heavy paper (Whatman paper) (Krasovsky et al., 2005a,b). Each series unites shells with the same thickness ratio $R / h=183 ; 245 ; 304 ; 452$ (Fig. 5a). Within each series, three specimens with the nominal value of the slope angle of the generator line to the shell base plane $\alpha=4^{\circ}, 6^{\circ}, 8^{\circ}, 10^{\circ}, 12^{\circ}, 15^{\circ}, 20^{\circ}$ and $25^{\circ}$ (Fig. 5a) were produced.

The production technology of samples and their preparation for testing includes marking and receiving the slab, making the actual sample and fastening it to the test fixture. The blank is a flat sweep cone (circle sector) with two allowances. The first allowance (width 1.5-3 mm) is designed to form an adhesive "overlapping" bond located along the cone. The second allowance (width $9-18 \mathrm{~mm}$ ) is designed to fix the test specimen in the fixture. The workpiece on the sheet is oriented (when was marked) in such a way that the main orthotropic direction of paper with minimal elastic and strength characteristics coincides with the axis of symmetry of the cone sweep. Tests were carried out for shells of all series under the same conditions of their attachment, which was the complete restriction to movement of the linear base of the cone edge.

Drawing paper of the brand "B" (GOST 597-73) was used for the production of samples. This material is best suited to the goals and objectives of the experiment. The efficiency of its use in the experimental study of stability of shells was proved in (Mossakovsky et al., 1989; Prokopalo, 1988, 2003).

Drawing paper has very high stability and uniformity of elasticity indicators, strength and plate geometry, despite of its expressed but minor orthotropic mechanical properties. Relative elastic characteristics (ratio of tensile strength to modulus of elasticity) are higher than the high-strength steel and other metals, which extends in the experiment the possible elastic deformation stage.

The main mechanical properties of the shell material are (Prokopalo, 2003): Young's modulus $E_{x}=6.9 \cdot 10^{3} \mathrm{MPa}, E_{y}=3.4 \cdot 10^{3} \mathrm{MPa}$, shear modulus $G=1.92 \cdot 10^{3} \mathrm{MPa}$, Poisson's ratio $\nu_{x}=0.3, \nu_{y}=0.15$, ultimate strength $\sigma_{x}=45 \mathrm{MPa}, \sigma_{y}=31 \mathrm{MPa}$ (here indices $x, y$ correspond to the main directions of orthotropic properties of the paper sheet). Four material properties are given due to the fact that only linear elastic strain is presented during the experimental process. Thickness of sheet was $h=0.23 \mathrm{~mm}$. This value was constant for all paper sheets. Sheet density was $200 \mathrm{~g} / \mathrm{m}^{2}$. Thus, all specimens had the same thickness $h=0.23 \mathrm{~mm}$ and 4 different radii of the cone base whose value for series $1,2,3$ and 4 was $R=42,56.25,70$ and $104 \mathrm{~mm}$, respectively.

The shell was folded by a label and fixed in position with glue BF2 that was applied according to markings on the mating faces of sweep and the allowance. After bonding, the compound was pressurized for 12 hours by two narrow rectilinear plates.

The allowance components of the sample from the base of the cone was cut along the generator line (in order to implement the necessary conditions for fixing), and was folded to the level of the plane of the shell base, and then stuck by the glue BF2 flat end surface to the test fixture. The outer side of the allowance element compound was filled with glue and glued to it by a retaining ring (to ensure tightness), cut from cardboard, $1 \mathrm{~mm}$ thick. The inner diameter of the ring corresponded to the outer diameter of the cone at its base. The resulting fixation was pressurized during 12 hours by clamping devices to the end ring over its entire surface. Resulting fixation completely restricted three linear movements and partially rotation angles of edge-section and corresponded to elastic clamped support, which lay between the canonical boundary conditions of the fixed hinge and fixed clamped support.

The height $H$ of each sample was measured with an accuracy of $0.01 \mathrm{~mm}$ (dial indicator movement) after fixing it to the test device that was determined on the basis of the actual $\alpha$ value and then was used later in the calculation and analysis of the research results. 
Two series of shells with different angles $\alpha$, fixed on the flange of the test device, are presented in Fig. 1.

(a)

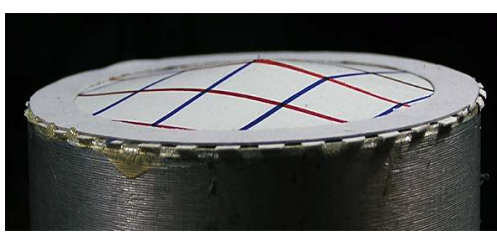

(b)

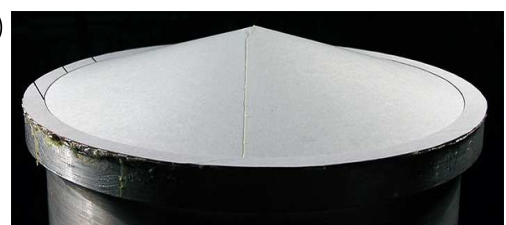

Fig. 1. Shells with $\alpha \approx 4^{\circ}$ (a) (series 1 ) and $\alpha \approx 20^{\circ}$ (b) (series 4 ) fixed on the flange of the test device

Glue line connecting the sample retaining ring and the flange of the test device is clearly visible. The test device is a metallic hollow circular cylinder with a hermetically closed lower end and the upper end of a free-flange, to which the sample plane is attached. The inner cylinder diameter corresponds to the base of a conical shell $2 R$ and to width of the flange - bandwidth allowance providing fixation sample. Cylinder height depending on $R$ was from $200 \mathrm{~mm}$ (small values of $R$ ) to $350 \mathrm{~mm}$.

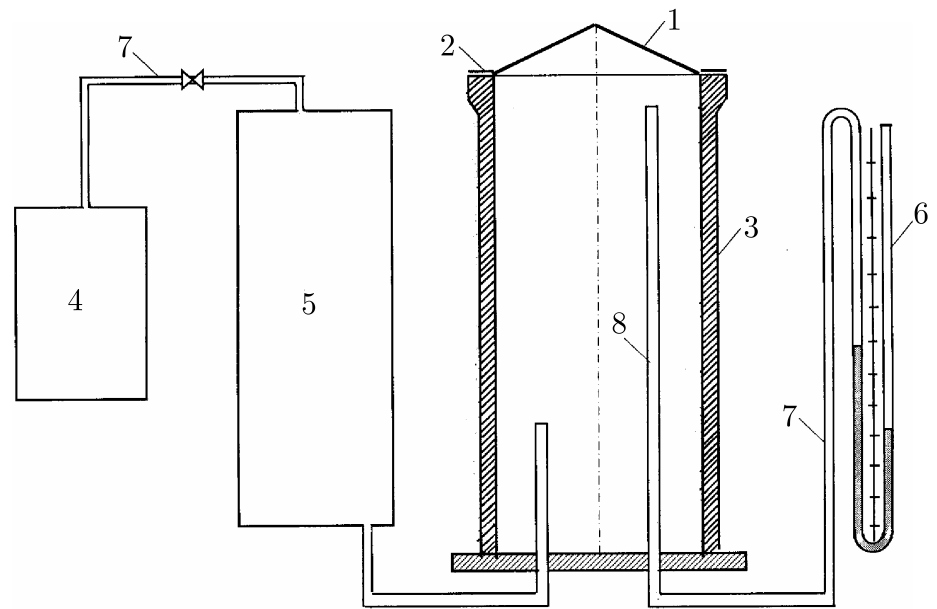

Fig. 2. Scheme the of test: 1 - specimen, 2 - fixing ring, 3 - testing device, 4 - vacuum pump, 5 - receiver, water manometer, 7 - flexible hoses, 8 - manometer branch pipe

The loading of the samples was carried out by external air pressure (by vacuuming the inside of the cavity of the shell). The scheme of tests is shown in Fig. 2. Evacuation was carried out by a vacuum pump that, in order to ensure a smooth change in pressure, was connected through a large receiver $\left(\sim 50 \mathrm{dm}^{3}\right.$ volume) with the cavity of the test device that played the role of a small receiver (depending on $R$ varying from $\sim 1 \mathrm{dm}^{3}$ to $\sim 12 \mathrm{dm}^{3}$ ), and helped to maintain the pressure during shell buckling.

The change of pressure in the cavity of the small receiver that corresponded to the external pressure acting on the shell was measured by U-shaped water manometer with accuracy up to $10 \mathrm{~Pa}$. The loading process was carried out at a rate of about $1 \mathrm{~Pa} / \mathrm{s}$. The registration of buckling was performed visually.

Features of shell buckling were determined by their degree of flatness. The cones of all 4 series, similar to the plate (for values of $\alpha \approx 4^{\circ}$ ), lost their stability by drastic (single clap) jumps to the axial symmetric noncontiguous equilibrium form that, except for the edge area, consists of the original mirror image form relative to the base plane. Typical non-adjacent buckling forms are shown in Fig. 3. Accumulation and development of supercritical dents was accompanied by the emergence and growth of plastic deformation for series of samples 2-4 too.

At the same time, rotation of the end section of the shell was presented, indicating that the test implemented in the boundary conditions did not meet the rigid but elastic fixed clamped support. 

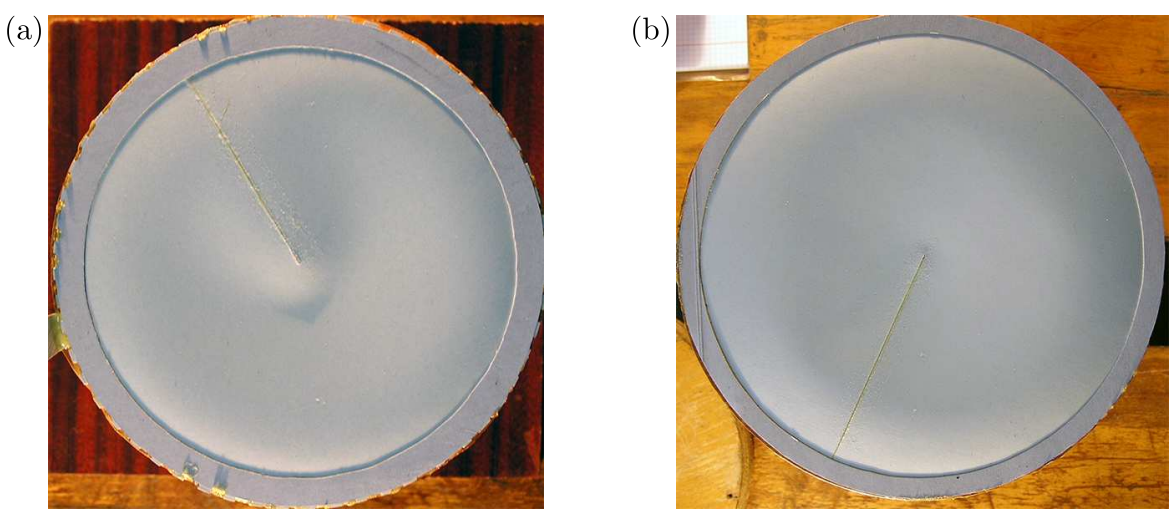

Fig. 3. Shells of series 3 (a) and 4 (b) with $\alpha \approx 4^{\circ}$ loss of stability by a single clap

Buckling (for $\alpha>6^{\circ}$ ) occurred in the circumferential direction in forn of periodically spaced indentations and bulges outside along with a half-wave generator. The buckling process was usually stretched in time. At first (soft stage), one or two or three small pits were formed without changing the pressure with centers located at the level of one third of the base of the cone. Developing deformations in samples of series 2-4 took place in the elastic stage of the work of the material, as evidenced by the results of the unloading (until complete recovery of the original shape) and the subsequent loading of the samples. Samples of series 1 had plastic deformations that were developed due to bending in the area of compression. The size of the first dents increased when the pressure increased, at the same time new dents arose, up to formation in the circumferential direction periodic supercritical configurations consisting of dents and bulges.

The value of pressure, when accumulation of dents occurred (from the first supercritical dents to their closed chains), was determined by the degree of flatness of the shell. The minimum pressure increased (2-5\%) in line for very shallow shells with $\alpha<10^{\circ}$, the maximum (20-25\%) - for specimen with $\alpha \sim 25^{\circ}$. Supercritical dents form of closed circular shells for series 2 are shown in Fig. 4.

(a)

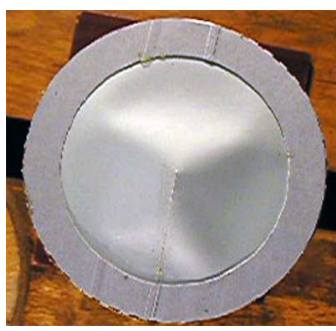

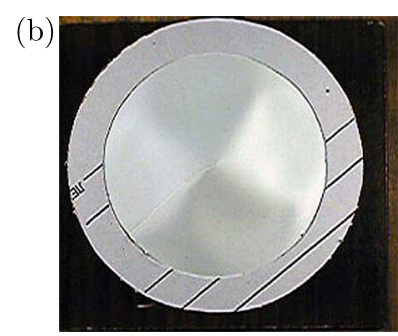

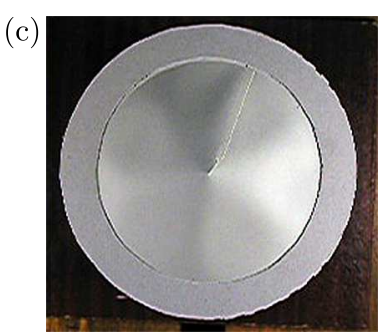

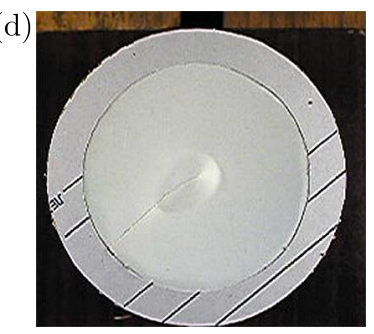

Fig. 4. Buckling forms of shells with: (a) $\alpha \sim 6^{\circ}$, (b) $\alpha \sim 12^{\circ}$, (c) $\alpha \sim 20^{\circ}$, (d) invert

All shells with $\alpha>6^{\circ}$ lost their stability except for a few samples whose buckling was occurred immediately by a single clap with forming of closed circular dents. Buckling load values of these samples were the highest between shells with the same nominal geometry.

The role of the connecting joint in the buckling process should be noted. Obviously, the seam breaks the symmetry of geometrical rigidity of shell. On the one hand, it increases the radial stiffness of the adjacent portion of the shell, on the other "suggests" additional initial imperfections. All this affects, in principle, subcritical deformation of the shell and, as a consequence, the critical pressure. However, this influence is manifested in a relatively narrow region adjacent to the weld. Since almost all the trials of the first generation of supercritical dents occurred in areas remote from the seam, it is believed that the joint impact on the value of the pressure at which the image of the first supercritical dents, is negligible. 
Increasing the pressure in the supercritical stage (after formation of the closed circuit dents) results an increase in the size of deflections and dents in the circumferential and radial directions. This curvature of the shell in the outer bulges increased as a result of which the latter were transformed into ribs delimiting dents. The subsequent increase in pressure leads to a rotationally symmetric inverted buckling form (very shallow shells with $\alpha \leqslant 8^{\circ}$ ), which took place, as a rule, by a clap or by restructuring to the supercritical form with changes in the number of dents, which also ends by an axially symmetric inverted form. And, if for very shallow shells supercritical axially symmetric configuration was close to the mirror image of the original form, the samples with $H /(2 R)>0.10\left(\alpha>12^{\circ}\right)$ inverted form was not complete: the area at the top of the cone was not to turn out. In these samples, at the boundary between the non-deformed central portion and turned inside the area formed a narrow area with a large radial curvature (rib). Due to the presence of edges connecting the seam, the plane shape differred from circular and has been elongated in the weld direction (Fig. 4d). The minimum transverse dimension of the non-deformed central portion with increasing $H /(2 R)$ also increased and amounted at $0.4 R$ from $H /(2 R) \approx 0.1\left(\alpha \approx 12^{\circ}\right)$ up to $0.9 R$ for $H /(2 R) \approx 0.23\left(\alpha \approx 25^{\circ}\right)$.

The value of the pressure at which there is a first supercritical dent was assumed as the experimental critical load $q_{\text {exp }}^{c r}$ in the case of axially non-symmetric buckling. As previously mentioned, the value of the pressure at which dents formed a closed circuit $q_{e x p, c}^{c r}$, was higher than $q_{\text {exp }}^{c r}$. For $\alpha<8^{\circ}$ the contrast values $q_{e x p}^{c r}$ and $q_{e x p, c}^{c r}$ were usually small (no more than $(1-2) \%)$; when $8<\alpha<12^{\circ}$ increased to $(5-10) \%$. At the same time, due to the small difference in values $q_{e x p}^{c r}$ and $q_{e x p, c}^{c r}$ specific values $q_{e x p, c}^{c r}$ were not recorded. For $\alpha>15^{\circ}$, the difference between $q_{e x p}^{c r}$ and $q_{e x p, c}^{c r}$ increased. In this aspect, control was made for values $q_{e x p, c}^{c r}$. It should be noted that in a group of test membranes with the same nominal values of $\alpha$, the minimum value $q_{e x p}^{c r}$ led to a supercritical form with a dent, and the maximum - the maximum configuration with a large number of dents.

\section{Comparison with existing theories}

The comparison of the critical pressure value obtained in the experiment $q_{e x p}^{c r}$ with the calculated pressure values $q_{c a l c}^{c r}$ of the linear (bifurcational) buckling problem considering linear subcritical straining was carried out by using ANSYS 7.1 software. Taking into account linear subcritical straining has given minimun calculated buckling load values. The limit pressure of geometrically nonlinear problem of the tested shells was higher than the critical pressure of the linear problem. Additionally, comparisons with calculations based on other approaches and theories (Krasovsky et al., 2005a,b) have been done.

In these studies, calculation of the geometric model of a closed conical shell was built using the method of "Rising simulation" on the "default" 4 conical panels $(k=4)$ with Coons patch surfaces. In this way, calculation of a non-perfect shell and a shell with initial geometry deviations meets geometry of the actual shell assembled from panels (Coones, 1967; Karasev, 2016).

Surfaces of the perfect and imperfect $(k=4)$ shells match at the base, and by 4 generator lines join the panel of the non-ideal shell, including the top. The rest of surfaces are characterized by imperfect shell portions at which the curvature in the circumferential direction is less than in circular (cross-section in the form of curvilinear squares) with the greatest deviation from the ideal surface of a circular cone in average the radial section panel. For the imperfect shell on the main part, it is characterized by a slight negative Gaussian curvature.

The technique of construction and features of such surfaces with deviations of the middle surface geometry, were described in detail in (Coones, 1967; Karasev, 2016).

Note that the highest relative and absolute amplitude in the deflection for the calculation model of the tested shells (shell series 4 with $R / h=452, \alpha=25^{\circ}, R=104 \mathrm{~mm}$ ) was 
$w_{\max }=14.9 \mathrm{~h}=3.43 \mathrm{~mm}$. This is a sufficiently large deviation of geometry comparing to the dimensions of the considered shell that is visually observed on the real object. In the experiments, unfortunately, the measurement of the initial geometrical imperfections was not carried out, but according to the visual inspection, the quality of the samples was reduced for the increasing index $R / h$, which in principle corresponds to a change in the quality of computational models. It is natural that such deviations from the ideal geometry of the forms led to a certain decrease in the estimated critical pressure. Calculated values of the relative critical load are presented in Table 1 for the considered four series of shells $\bar{q}_{i m p}^{c r}$ reflecting the maximum reduction in the critical pressure due to geometry deviation $(k=4)$. The values of $\bar{q}_{i m p}^{c r}$ are defined as the estimated critical pressure for the imperfect $q_{i m p}^{c r}$ and the ideal $q^{c r}$ conical shells with the same boundary conditions $\bar{q}_{i m p}^{c r}=q_{i m p}^{c r} / q^{c r}$.

It is seen from Table 1 that the initial geometry deviations lead to a reduction of $24 \%$ to $33 \%$ of the critical load.

Table 1. Minimum values of the relative critical load $\bar{q}_{i m p}^{c r}$ for imperfect $(k=4)$ shells of series $1-4(R / h=183-452)$

\begin{tabular}{|l|c|c|c|c|}
\hline \multicolumn{1}{|c|}{ Boundary conditions } & Series 1 & Series 2 & Series 3 & Series 4 \\
\hline \hline Fixed rigid clamped support & 0.682 & 0.667 & 0.730 & 0.692 \\
\hline Fixed hinge support & 0.734 & 0.682 & 0.762 & 0.702 \\
\hline
\end{tabular}

Note that there was a comparison (Krasovsky et al., 2005b) of the experimental data with the results of well-known theoretical research by Seyd P. (Grigolyuk and Kabanov, 1978) and Trapezin I.I. (Volmir, 1967). That comparison showed a good agreement with experiments only for non-shallow shells with $\alpha>20^{\circ}$. There was also a comparison with linear solutions given in the monograph of Karmishin et al. (1975). That comparison showed that calculation conducted throughout the considered range of the alpha in the ANSYS 7.1 software gave the best results comparing to experimental data.

\section{Calculation methods}

Numerical analysis of the influence of surface deviations of the median and orthotropic material resistance was carried out in ANSYS software (ANSYS Inc. Academic Research, Mechanical Analysis, Release 13.0 customer 298728) with the use of quadrangular SHELL 181 elements that had 4 nodal points (nodes located in the middle plane), each with six degrees of freedom. Such elements are suitable for calculation of thin-walled shells and for solving linear and nonlinear problems with large deformations and rotations. The elements have linear elastic properties. Isotropic, orthotropic and anisotropic elastic materials may be used as well.

(a)

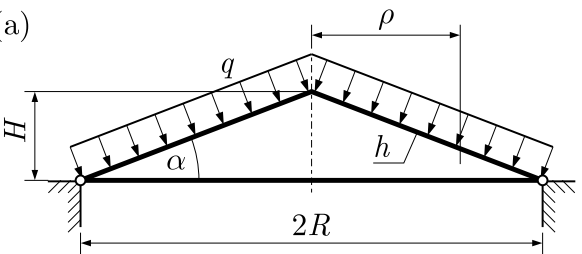

(b)

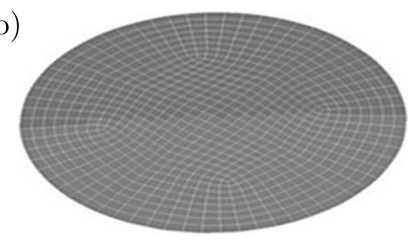

Fig. 5. Design scheme (a) and finite-elements model (b) of a closed shallow conical shell under external pressure

The loading was realized as an external transverse pressure $q$ uniformly distributed over the entire surface of the cone (Fig. 5). Boundary conditions of the base of the cone edge corresponded to a fixed clamped support and fixed hinge support. The calculations determined the critical 
pressure value of $q^{c r}$, and the buckling linear analysis was based on linear geometric subcritical deformation, in particular with a number of waves in the circumferential direction (the solution of the linear problem of stability).

The mesh partitioning process in ANSYS FE of the existing geometric model is a difficult task and is directly connected with the choice of the type of calculation and the nature of the decision. The mesh was built as a regular rectangular in plane. The size of the finite element was consistent with the recommendations given in the documentation of ANSYS (Release 13.0). At the early stages of calculation, the number $N$ of FE shells was taken close to 600 . In order to obtain reliable results, the net was successively thickened by approximately doubling $N$. The final calculations were carried out with $N$, which when increased by half, led to critical changes in the pressure by no more than $0.7 \%$ (when the critical dependence of the pressure on $N$ had stabilized). Thus, depending on the values of $R / h$ and the parameters $\alpha$ and $R$ in the final calculations, it was assumed that $N=3800-6900$.

\section{Comparison with experiment}

As already mentioned, the paper sheets the test samples were manufactured from, are characterized by weak elastic orthotropic indicators. At the same time, in the study by Krasovsky et al. (2005a), all calculations of the critical pressure and sample forms of buckling were carried out without the orthotropic properties. The isotropic shells had averaged elastic constants, in particular the modulus material elasticity $E=5.15 \cdot 10^{3} \mathrm{MPa}$ and Poisson's ratio $\nu=0.225$. The difference of this study from the work by Krasovsky et al. (2005a) was taking into account orthotropic material properties.

It should be noted that strict consideration of orthotropic properties of the shell material while determining the critical pressure in ANSYS is a rather complicated task, since the convolution of orthotropic sheet directions of the principal curvatures of the shell does not coincide with the directions of orthotropic properties. Construction of the corresponding mesh of finite elements, without making serious errors in the solution of the problem, is also extremely difficult. At the same time, the characteristics of the buckling shell at the bifurcation were caused by external pressure (a lot of waves in the radial direction extending along the generator line). A quite satisfactory assessment of the influence of orthotropic properties on the critical pressure may serve as a model of a conical shell with the direction of material orthotropic properties in the direction of the principal curvatures. In this case, the reasons are the following. A conical shell from an orthotropic furled sheet is characterized by heterogeneity of mechanical properties of the material in the circumferential direction and in the direction of the generator line. However, in each of such shells there can be identified a sufficiently broad area in which the direction of orthotropic properties will be close to directions of the principal curvatures. It can be most likely assumed that this is the area where the shell initiates overall buckling.

In this case, two orientations of orthotropic properties were considered: the first - with a greater longitudinal stiffness, the second - with a greater circumferential stiffness. As a result of calculations, it was found that in both cases there was an orthotropic property orientation substantially similar to significant reduction of the critical pressure. For small angles $\alpha$, this reduction for all series was of the order of $6-8 \%$, for large - from $20 \%$ for $R / h=183$ to $13-15 \%$ for the other series. As an example, in Table 2, for $R / h=183$ samples of the four series $R / h=183,245,304,452$ with the minimum and maximum values of the slope angle $\alpha$ of the generator line for the fixed hinged support, the critical pressure $q^{c r}$ is given for two cases of orthotropic sheet orientations. In the first case, the greater rigidity direction coincides with the shell generator line $x$, and in the second with the guide (in the circumferential direction) $y$. 
Table 2. Values of the calculated critical pressure $q^{c r}[\mathrm{kPa}]$ for shell series 1 and 4

\begin{tabular}{|l|c|c|c|c|c|c|c|c|}
\hline \multicolumn{1}{|c|}{$R / h$} & \multicolumn{2}{|c|}{183} & \multicolumn{2}{c|}{245} & \multicolumn{2}{c|}{304} & \multicolumn{2}{c|}{452} \\
\hline \hline$\alpha$ & $3^{\circ} 45^{\prime}$ & $26^{\circ} 34^{\prime}$ & $3^{\circ} 33^{\prime}$ & $24^{\circ} 8^{\prime}$ & $3^{\circ} 55^{\prime}$ & $26^{\circ} 8^{\prime}$ & $3^{\circ} 21^{\prime}$ & $26^{\circ} 8^{\prime}$ \\
\hline Isotropic & 0.528 & 11.18 & 0.241 & 4.678 & 0.151 & 3.088 & 0.048 & 1.200 \\
\hline Orthotropic $(x)$ & 0.495 & 8.869 & 0.224 & 4.053 & 0.139 & 2.670 & 0.045 & 1.024 \\
\hline Orthotropic $(y)$ & 0.491 & 8.903 & 0.222 & 4.061 & 0.139 & 2.681 & 0.045 & 1.028 \\
\hline
\end{tabular}

The experimental and computational critical pressures are presented in Figs. 6a-6d, for the angle $\alpha$ of the generator line for shell series 1-4.

(a)

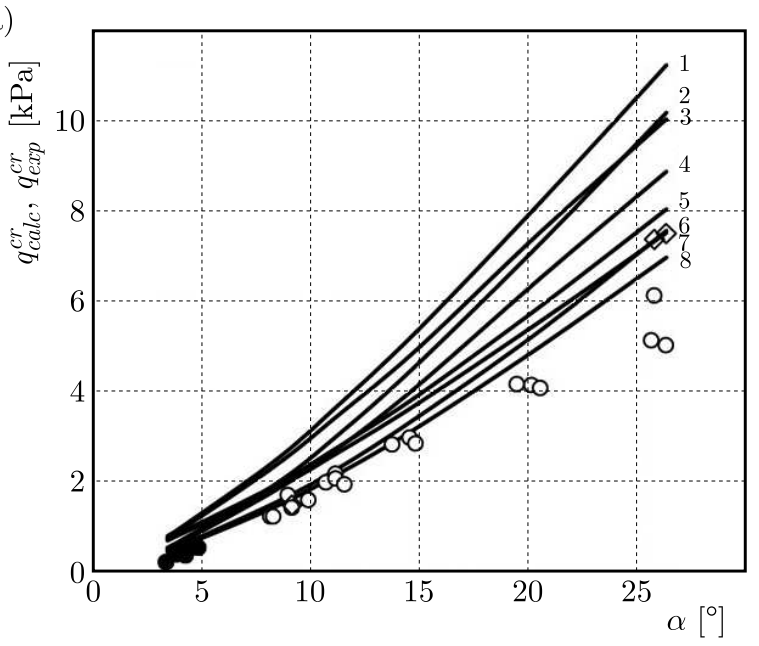

(c)

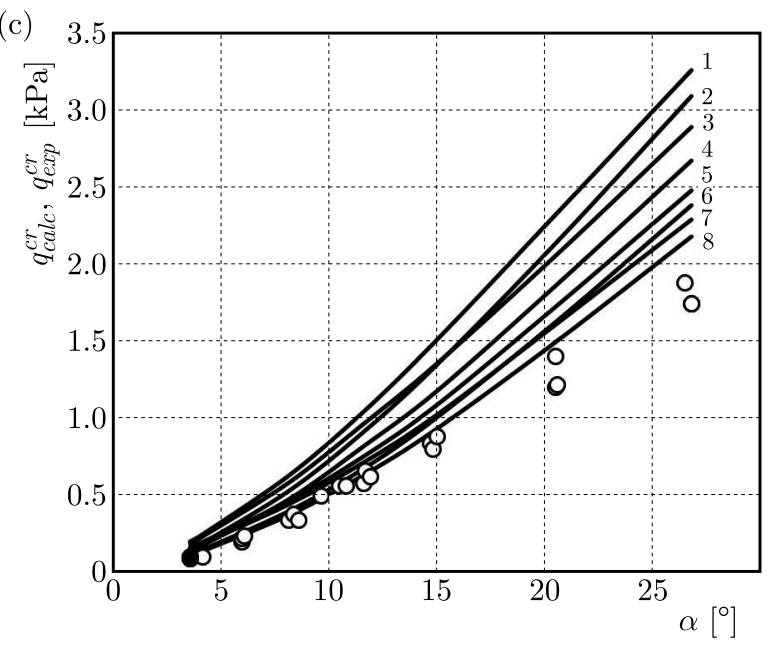

(b)

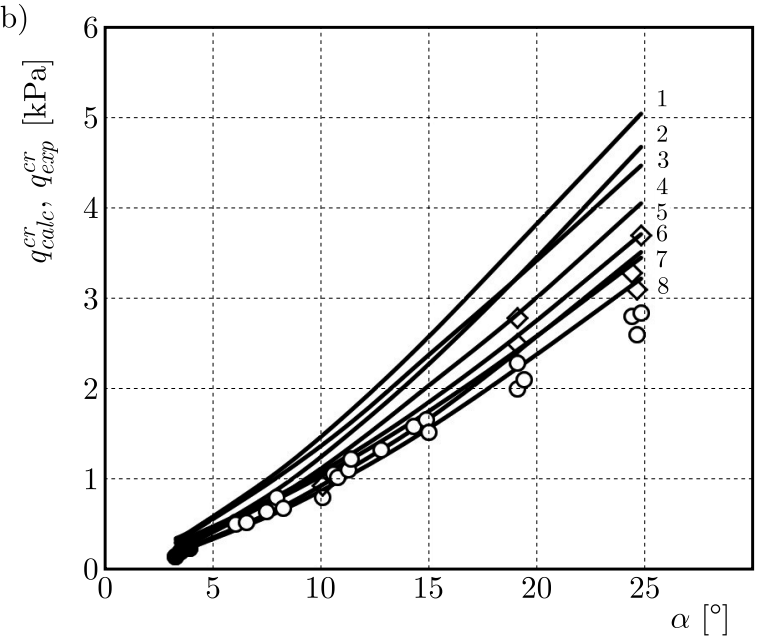

(d)

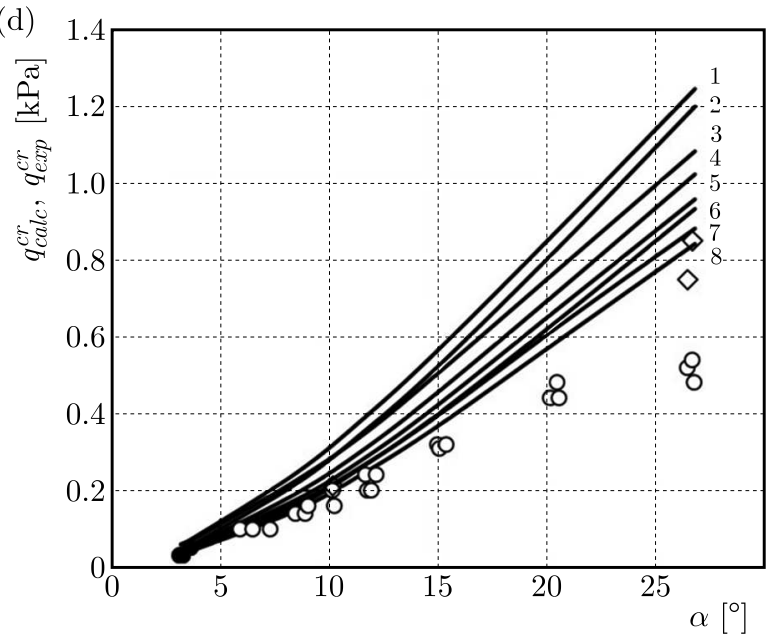

Fig. 6. Experimental and calculated dependencies of critical pressures on the angle $\alpha$ for shells: (a) series 1, (b) series 2, (c) series 3 , (d) series $4(R / h=183, R=42 \mathrm{~mm})$

Here, white circles and rhombus match the experimental values of the critical buckling pressure for the asymmetric, whereas dark circles - for the axially symmetric inverted form. The calculated curves with even numbers correspond to the fixed hinge support edge of the shell, with the odd - to the fixed clamped support. Curves 1 and 2 represent the critical pressure for geometrically perfect isotropic conical shells with averaged elastic constants, curves $3,4-$ for geometrically perfectly orthotropic shells, curves 5, 6 - imperfect shells from an isotropic material with averaged properties forming Coons patch surfaces of four panels, curves 7, 8 imperfect (Coons 4 panels) orthotropic shells. 
From those relationships it can be seen that the best agreement with the experimental samples of all series is obtained for shells with fixed rigidly clamped edges, with deviations in geometry and material orthotropic properties taken into account. Note that the dependencies are linear for $\alpha \geqslant 10^{\circ}$, either for the experimental data or obtained from the numerical analysis.

\section{Conclusions}

Comparison with the known extensive test results of elastic flat closed conical shells made of sheets of paper has shown that from a wide variety of theories and computational methods, including those refined, the best agreement with the experiment was achieved for calculation made in ANSYS software by taking into account periodic initial geometry deviations in the circumferential direction and a weak orthotrops of the shell material.

Thus, the critical pressure of existing conical shells with known forms of deviation from the ideal shape can be obtained in ANSYS by introducing and taking into account all deviations of the structure features, characteristics of the material as well as initial imperfections and other design scheme disturbances in the geometrical and calculated shell models.

\section{Acknowledgment}

This work was supported by the Alexander von Humboldt Foundation (Institutional Academic Co-Operation Program) (grant No. 3.4-Fokoop-UKR/1070297).

\section{References}

1. ANSYS Inc. Academic Research, Release 13.0, Help System, Mechanical Analysis Guide

2. Bushnell D., 1981, Buckling of shells-pitfall for designers, AIAA Journal, 19, 9, 1183-1226

3. Coons S., 1967, Surfaces for computer aided design of space forms. Technical report, MIT

4. Elishakoff I., 2012, Probabilistic resolution of the twentieth century conundrum in elastic stability, Thin-Walled Structures, 59, 35-57

5. Grigolyuk E., Kabanov V., 1978, Stability of Shells (in Russian), Science, Moscow

6. Hoff N.J., Singer J., 1960, Buckling of circular conical shells under external pressure, Proceedings of IUTAM Symposium of the Theory of Thin Elastic Shells, North Holland Publ. c.o., 389-414

7. KARASEv A.G., 2016, Initial imperfection influence on the buckling load of closed elastic isotropic shallow conical shells, Mathematics and Mechanics of Solids, 21, 4, 444-453, DOI: $10.1177 / 1081286514526082$

8. Karmishin A.V., Lyaskovets V.A., Myachenkov V.I., Frolov A.N., 1975, Statics and Dynamics of Thin-Walled Shell Structures (in Russian), Engineering Industry, Moscow

9. Krasovsky V., Karasev A., 2016, Properties of numerical solution of the deformation and stability problem in shallow conical shells under external pressure, Roads and Bridges - Drogi i Mosty, 15, 117-135, DOI: 10.7409/rabdim.016.008

10. Krasovsky V., Prokopalo E., Varyanichko M., 2005a, Buckling of shallow conical shells under external pressure in physical and numerical experimets (in Russian), News of Science of Pridniprov'ya, Series "Engineer sciences", 2, 20-31

11. Krasovsky V., Prokopalo E., Varyanichko, M., 2005b, Experimental and theoretical study of closed shallow conical shells buckling under external pressure (in Russian), Theoretical Foundations of Civil Engineering, Polish-Ukrainian Transactions, Dnepropetrovsk, 13, 175-188

12. MaAli M., Showkati H., Fatemi S.M., 2012, Investigation of the buckling behavior of conical shells under weld-induced imperfections, Thin-Walled Structures, 57, 13-24 
13. Mossakovsky V.I., Obodan N.I., Prokopalo E.F., 1989, Influence of geometrical nonlinearity on the destruction of thin-walled structures with ruptures (in Russian), Report Academy of Science USSR, 308, 5, 1079-1081

14. Prokopalo E.F., 1988, Experimental research of cylindrical shells buckling undergoing external linearly varying along the generatrix pressure (in Russian), Structural Mechanics and Calculation of Facilities, 6, 61-64

15. Prokopalo E.F., 2003, Stability of circular cylindrical panels under axial compression (in Russian), Theoretical Foundations of Civil Engineering, 11, 227-230

16. SEIDE P., 1962, A survey of buckling theory and experiment for circular conical shells of constant thickness, NASA TN D-1510, 401-426

17. Singer J., 1961, Buckling of circular conical shells under axisymmetrical external pressure, International Journal of Mechanic Science, 4, 3, 330-339

18. Volmir A.S., 1967, Stability of Deformable Systems (in Russian), Science, Moscow

Manuscript received June 17, 2019; accepted for print July 17, 2019 